\title{
3D Homogenisation of concentrated windings with rectangular conductors
}

\author{
P. Romanazzi ${ }^{1}$, S. Ayat ${ }^{2,3}$, R. Wrobel ${ }^{2}$, Senior Member, IEEE, D. A. Howey ${ }^{1}$, Member, IEEE \\ ${ }^{1}$ Department of Engineering Science, University of Oxford, Oxford, UK, pietro.romanazzi@eng.ox.ac.uk \\ ${ }^{2}$ Department of Electrical and Electronic Engineering University of Bristol, Bristol, UK \\ ${ }^{3}$ Motor Design Ltd., Ellesmere, UK
}

\begin{abstract}
Design optimisation of electromagnetic devices is strongly affected by thermal behaviour. In order to check whether thermal limitations are exceeded, one has to accurately evaluate the loss generated for each working condition and feed it to a thermal model. Using the two models in sequential manner, however, can lead to an error in the loss estimation. Moreover, a proper loss distribution calculation allows for a more precise temperature field computation, allowing for an accurate prediction of the hot-spot temperature. When $\mathrm{AC}$ effects are significant, this process becomes more computationally expensive since every single wire should be included in the model and this inevitably slows the design procedure. In this work a method is presented for model order reduction of coupled 3D electromagnetic-thermal finite element analysis via homogenisation. The method, applied to an hardware exemplar representative of an open-slot modular stator-winding assembly, is demonstrated to provide very accurate results compared to a fine model where every single conductor is taken into account, but with a significant reduction in simulation time. Extensive experimental validation is also provided. Due to the reduction in computational effort, the method is suitable for improving the design process and reducing the time to market of many electromagnetic devices, including electrical machines, inductors and transformers.
\end{abstract}

Keywords-Homogenisation, AC losses, loss separation, thermal analysis, electrical machines

\section{INTRODUCTION}

During the development of new electromagnetic devices, such as machines, transformers or inductors, it is very important to accurately evaluate the losses generated during operation. In particular, it is essential to evaluate the loss distribution within every component at each working condition. For instance, at high frequencies, in addition to the Joule effect, the windings experience losses due to $\mathrm{AC}$ effects, i.e. skin and proximity effect. The magnitude of these depends on a number of factors, such as wire shape (circular, rectangular, etc.), wire dimension, filling ratio, the specific pattern of the magnetic flux, and temperature. Detailed knowledge of the loss distribution, coupled with an accurate thermal model, allows for a precise estimation of performance, efficiency and temperatures. This can lead to an optimised design with reduced cost.

This work was supported by the European Union: FP7 ITN Project 607361 ADEPT
The thermal model accuracy mainly depends on three factors: (i) model formulation (based on the finite element (FE) method or lumped parameter thermal networks (LPTN)) [1], (ii) empirical material data and build factors [2]-[4] and (iii) loss distribution estimation [2,4,5]. The focus of this work is on the latter, and in particular on computationally efficient estimation of loss and temperature distribution within the windings, which are in most cases the limiting factor in the design. In particular, the goal is to effectively evaluate the temperature dependence of the loss when $\mathrm{AC}$ effects are present and the resulting hot-spot temperature.

In the literature, the most common methods to capture loss distribution including AC effects, use analytical approaches [6,7] or fine FE models where every single wire is taken in account $[2,4,8,9]$. The former are limited to $2 \mathrm{D}$ domains and simple geometries. The latter can be frequently computationally prohibitive, in particular when 3D simulations are involved. The solution to dramatically reduce the computational cost of FE simulations is to homogenise the winding domain, replacing the complex structure of conductors with an homogenised material with equivalent material properties, for the electromagnetic and thermal problems. This process is sketched in Fig. 1, where the detailed winding of the fine model is replaced with an homogenised winding.

In the literature a number of methods are available for the electromagnetic homogenisation of windings in the frequency domain using 2D FE [10]-[13]. Due to its straightforward implementation, in this work we employ and extend the method described in [10]. Compared to [14,15], here we show how to apply the method to a 3D domain with no axi-simmetry, including thermal effects; extensive experimental validation is also provided. The techniques is applied to an hardware exemplar of an open-slot modular stator-winding assembly with profiled conductors (from now on referred to as "motorette"), see Fig. 1. The objective is to evaluate the effectiveness of the model in predicting the loss distribution within the winding domain, accounting for the loss temperature dependence, and the resulting thermal field. This technique enables a factor 30 reduction in simulation time with the same level of accuracy. The match with the measurements on the motorette is also good, with the model slightly overestimating the loss $(\approx 20 \%$ discrepancy) and top temperature $\left(\Delta T<3{ }^{\circ} \mathrm{C}\right)$ 


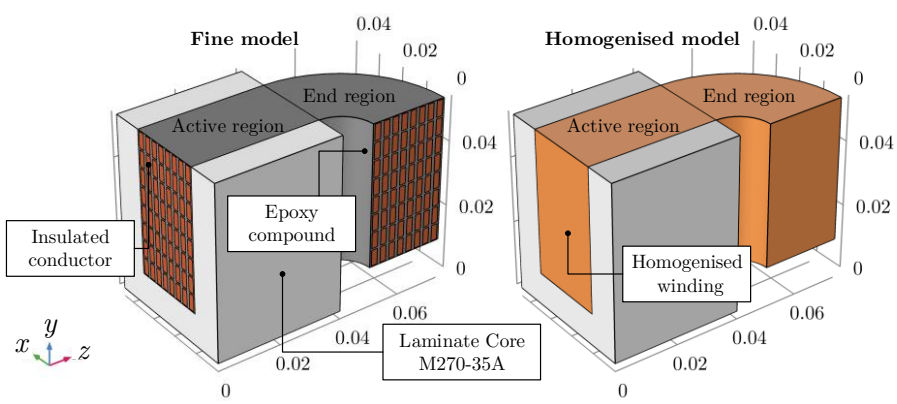

FIG. 1: 3D Model of the analysed specimen - one quarter of the whole motorette. Active winding total length $80 \mathrm{~mm}$, teeth height $44 \mathrm{~mm}$ and slot opening $24 \mathrm{~mm}$. Fine model (left) with 64 turns of insulated copper conductor $2 \times 5 \mathrm{~mm}$ with epoxy potting, and homogenised model (right)

\section{3D HOMOGENISATION}

\section{A. Electromagnetic parameter estiamtion}

To successfully homogenise the 3D winding domain, the conductors should be all connected in series and assumed to be periodically distributed, e.g. on a square lattice (see Fig. 1), in the slot. If these conditions are respected, the effective material properties of the homogeneous material can be obtained from the study of so-called "cell problems", that is obtaining $\mathrm{M}(\omega)$ $[11,12]$

$$
\{\boldsymbol{H}, \boldsymbol{E}\}=[\mathbf{M}(\omega)]\{\boldsymbol{B}, \boldsymbol{J}\}
$$

For the specific case of centrosymmetric conductors, the matrix $[\mathbf{M}(\omega)]$ is block diagonal, with the blocks consisting of the effective reluctivity $\left[\nu_{e q}(\omega)\right]$ and the effective resistivity $\left[\rho_{e q}(\omega)\right]$, respectively, where $\omega=2 f \pi$ with $f$ frequency. As shown in [10], the cell problem is solved in $2 \mathrm{D}$, accounting for the wire shape, distribution and filling ratio $\lambda$, defined as $\lambda=$ $\frac{n_{w} A_{w}}{A_{s l o t}}$ with $n_{w}$ number of conductors and, $A_{w}$ and $A_{\text {slot }}$ the conductor and slot cross-sectional area, respectively. Applying suitable boundary conditions, one is able to calculate $\left[\nu_{e q}(\omega)\right]$ and $\left[\rho_{e q}(\omega)\right]$ evaluating the complex power absorbed by the central cell when the proximity or skin effects are excited, respectively. For instance, in Fig. 2(a) we show the solution of the cell problem when a unit average induction at $X=0.6$ is imposed along the $x$ axis with 0 net current in the conductors (condition to excite the proximity effect only), showing the resulting current density distribution and flux lines, that allows to obtain $\left[\nu_{e q}(\omega)\right] ; X=\frac{r_{e q}}{\delta}$ is the reduced frequency, where we introduced the equivalent conductor radius $r_{e q}=\sqrt{A_{w} / \pi}$ and the skin depth $\delta=\sqrt{2 \rho / \omega \mu_{0}}$, with $\rho$, $\mu_{0}$ being the conductor electrical resistance and permeability of free space, respectively. From Fig. 2(a) one can also understand that for rectangular conductors the effective reluctivity is anisotropic.

According to [10], we can express the resulting equivalent properties in terms of dimensionless parameters $p$ and $q$, see Fig. 2(b)

$$
\begin{aligned}
\nu_{e q x, y} & =\nu_{0}\left(q_{B} x, y\right)+j \frac{\lambda r_{e q}^{2} \omega}{4 \rho}\left(p_{B x, y}\right) \\
Z_{e q} & =p_{I} R_{D C}+j q_{I} \omega \frac{\mu_{0} L}{8 \pi \lambda}
\end{aligned}
$$

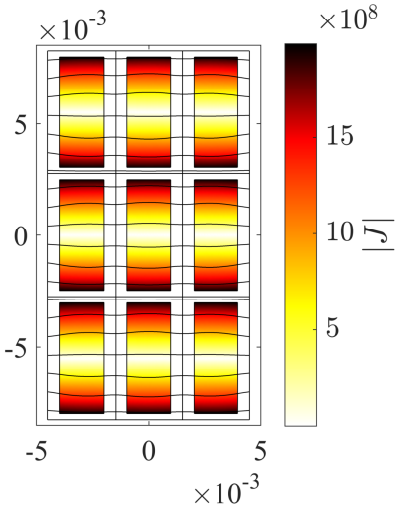

(a)

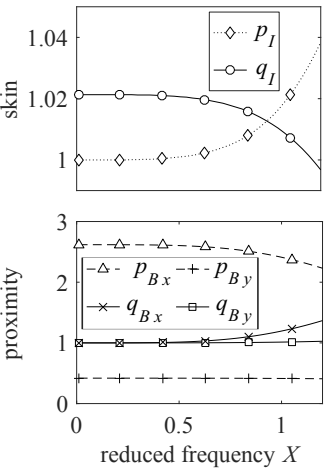

(b)
FIG. 2: (a) Solution of the proximity effect cell problem due to a unit average induction along $x$, showing the resulting module of the current density and flux lines. (b)Dimensionless parameters for (top) skin and (bottom) proximity effect for $2 \times 5 \mathrm{~mm}$ conductor with $\lambda=0.606$ (slot dimensions $24 \times 22 \mathrm{~mm}$ ) against reduced frequency

where $R_{D C}$ is the wire DC resistance and $L$ is the conductor length. It is important to notice that the effective resistivity results in an effective impedance $Z_{e q}$ to be included in the external electrical circuit equations. Moreover, as shown in [14], the parameters presented in Fig. 2(b) include a thermal effect if the temperature dependence of electrical conductivity is captured with the linearised formula $\rho(T)=\rho_{0}\left(1+\alpha\left(T-T_{0}\right)\right)$, with $\alpha$ the linear thermal parameter of case of conductors.

\section{B. Thermal parameter estimation}

The outcome of thermal homogenisation is the equivalent thermal conductivity $\left[k_{e q}\right]$ and heat capacity $C_{e q}$ of the winding domain. Although $C_{e q}$ can be simply computed by volume averaging the heat capacities of the various materials, a number of methods are available to obtain $\left[k_{e q}\right]$, either analytical, numerical or experimental [16]. In this work we exploit the multiple-scales (MS) method, as presented in [17], since it can efficiently evaluate the equivalent thermal features of the windings through the study of the specific cell problem with FE. The MS method includes the effect of $\lambda$ and wire shape (rectangular wire in this case). Following the MS method, the solution of the cell problem provides us with $k_{e q x}$ and $k_{e q} y$.

Although it is quite complicated to obtain the equivalent thermal conductivity in the $x$ and $y$ directions, it is easier to deal with the $z$ direction. In fact, as the wires lie in parallel along the $z$ direction, we can simply apply the higher bound of the Wiener formula, $k_{e q z}=\lambda_{c} k_{c}+\lambda_{i} k_{i}+\lambda_{e} k_{e}$, with $\lambda_{c}, \lambda_{i}$ and $\lambda_{e}$ being the conductor, insulation and epoxy filling ratio, respectively $\left(\lambda_{c}+\lambda_{i}+\lambda_{e}=1\right)$.

\section{Application to a $3 D$ domain}

The complexity of applying the method to 3D domain lays on the fact that the homogenised material is anisotropic. If we refer to the active winding part only with the reference coordinate system of Fig. 1, the equivalent anisotropic material 
properties, e.g. reluctivity and thermal conductivity, should be expressed as

$$
\left[\nu_{e q}\right]=\left[\begin{array}{ccc}
\nu_{e q} x & 0 & 0 \\
0 & \nu_{e q} y & 0 \\
0 & 0 & \nu_{0}
\end{array}\right]
$$

In the active region we can assume the problem to be $2 \mathrm{D}$, since the direction of the current is along the $z$ axis, (Fig. 1). Accordingly, in this region, we can assume the magnetic field to always be parallel to $x-y$ plane. This also means that the permeability along the $z$ direction should not include the effect of eddy currents [18] and can keep its real value $\nu_{0}$.

On the other hand, the anisotropic equivalent properties in the end-winding region can be evaluated at each angle using the elemental rotation matrix about the $z$ axis $\left[R_{z}\right]$, e.g. $\left[\nu_{e q}\right]_{e w}=\left[\nu_{e q}\right]\left[R_{z}\right]$, where the subscript ew stands for end-winding region. For more complex winding arrangements, suitable transformation matrices should be employed.

\section{RESUlts}

\section{A. Numerical validation}

The coupled 3D homogenisation technique presented here is now applied to the reference motorette described in Fig. 1. The domain consists of $n_{w}=64$ rectangular conductors connected in series and excited with sinusoidal current at various frequencies. The hardware demonstrator has been designed to purposely exaggerate the $\mathrm{AC}$ effects and better prove the performance of the proposed model. To reduce the mesh size of the full 3D problem, but without losing generality, the conductor insulation domain is neglected for both the electromagnetic and thermal problems. Also, at this initial stage, the build factors (i.e. thermal contact resistances), will not be included in the model. Regarding the thermal boundary conditions, a fixed temperature of $40^{\circ} \mathrm{C}$ is applied to the base, as if the specimen was placed on a cold plate, whereas convection with ambient is assumed to all the remaining free surfaces $\left(h_{a m b}=15 \mathrm{~W} /\left(\mathrm{m}^{2} \mathrm{~K}\right)\right.$ and $\left.T_{a m b}=40^{\circ} \mathrm{C}\right)$. The model considers the non-linear behaviour of the laminated core (M270-35A) and the losses are estimated using the modified Steinmetz equation with the parameters given in [19]. Radiation effects are neglected.

The results from the coupled electromagnetic and thermal homogenisation in the reference motorette in terms of steadystate winding loss estimation are presented in Fig. 3. Fig. 3(a) presents the total loss estimation obtained with the fine and homogenised model for different current amplitude and at growing frequency. The match between the models is very close, with a maximum relative error around $2 \%$. If we split the loss contribution from the active and the end winding, as shown in Fig. 3(b) for the case of $I_{r m s}=15 \mathrm{~A}$, we can see that the bigger discrepancy is concentrated in the active region. In the end of the active length of the the current, due to the $\mathrm{AC}$ effects, exhibits components along directions other than $z$ [19], reducing the validity of the 2D approximation. Moreover, the solution time for the coupled electromagneticthermal steady-state analysis on an Intel i7 $(3.2 \mathrm{GHz}$ and $32 \mathrm{~Gb}$

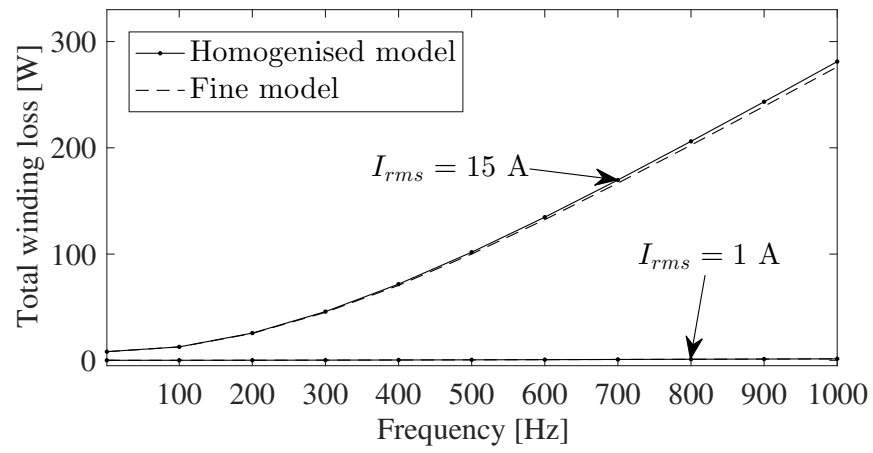

(a)

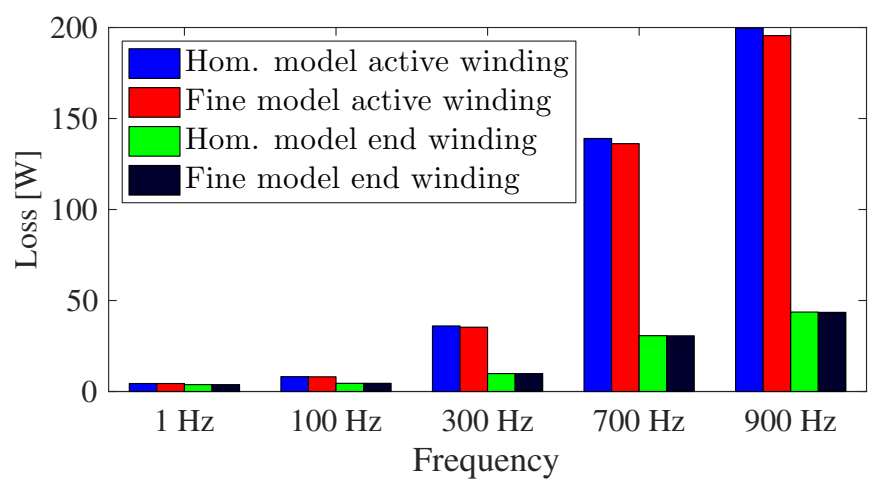

(b)

FIG. 3: Comparison between the fine and homogenised models in the estimation of (a) total loss for different frequencies and current amplitude ( $I_{r m s}$ is current rms value) and (b) loss split between active and end winding for the case of $I_{r m s}=15 \mathrm{~A}$

RAM) on average is around $2700 \mathrm{~s}$ vs. $96 \mathrm{~s}$ with the fine and homogenised models, respectively.

The coupled electromagnetic-thermal analysis enables to evaluate the temperature distribution within the domains, obtained by iteratively updating the thermal model with the loss distribution from the electromagnetic model. As an example, in Fig. 4 we present the loss and temperature distribution obtained with the fine and homogenised models, respectively, for the case of $I_{r m s}=15 \mathrm{~A}$ and $f=500 \mathrm{~Hz}$. It is important to notice that in Fig. 4(a), where the losses are plotted using a logarithmic scale, the homogenised model provides only with the cell averaged loss. This prevents to show the precise loss distribution resulting from the circulating eddy currents in each conductor. However, since the copper has a high thermal conductivity compared to the rest of the materials (see Table I), this doesn't affect the homogenised thermal model, which is able to give with high accuracy the temperature field, Fig. 4(b)

In Fig. 5(a) the hot-spot and mean temperature calculated with the fine and homogenised models are compared. The maximum temperature difference between the models is within $1{ }^{\circ} \mathrm{C}$ which is mainly due to the error in the loss estimation. It is interesting to notice how the mean temperature, shown only for the case of $I_{r m s}=15 \mathrm{~A}$, is about $25 \%$ lower than the hotspot at high frequencies.

The advantage of the coupled electromagnetic and thermal 

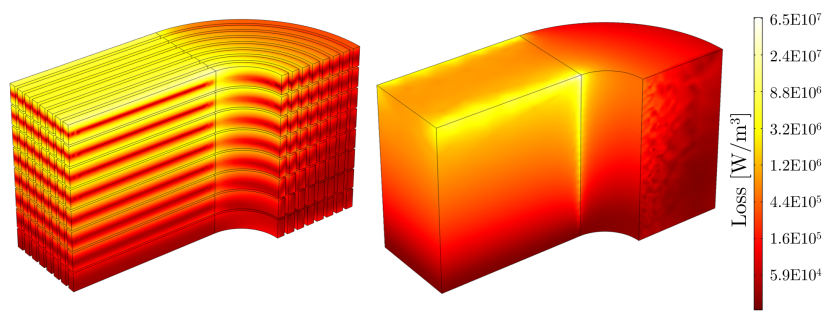

(a)
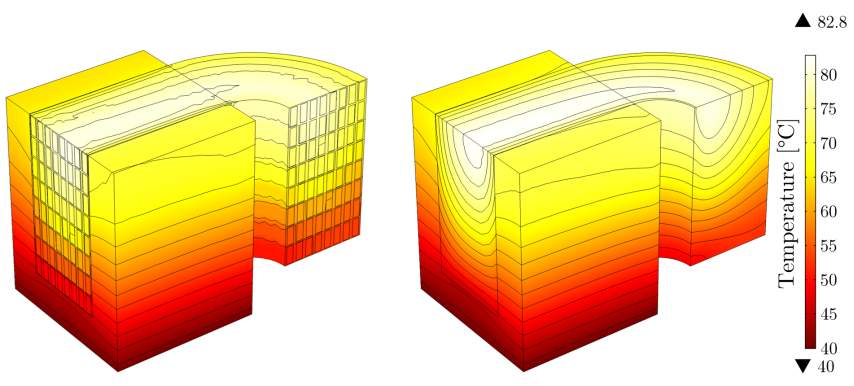

(b)

FIG. 4: Comparison between the fine and homogenised model in the estimation of the (a) loss and (b) temperature distribution within the motorette with $I_{r m s}=15 \mathrm{~A}$ and $f=500 \mathrm{~Hz}$

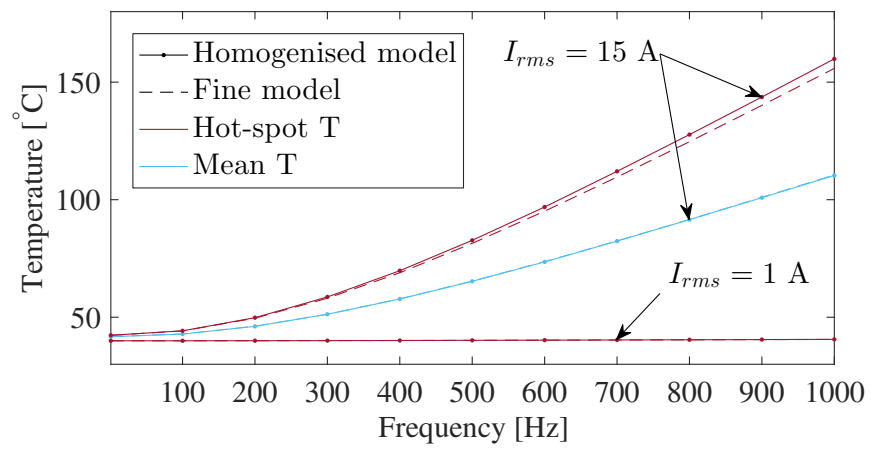

(a)

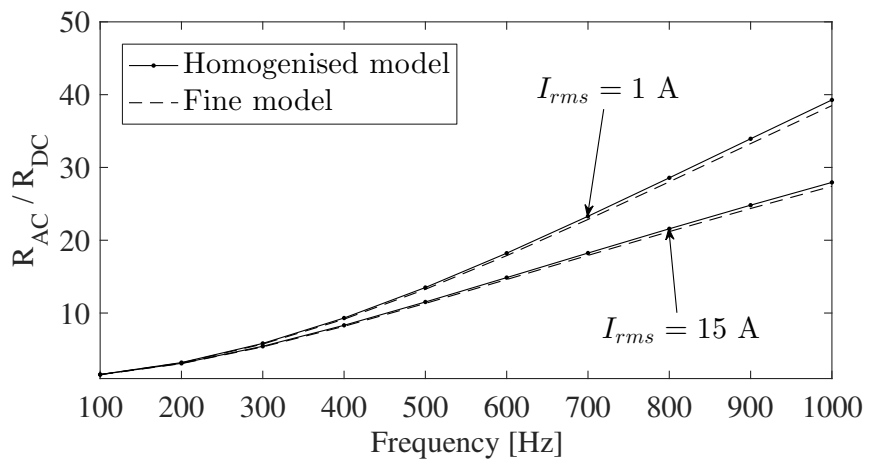

(b)

FIG. 5: (a) Winding hot-spot (dark line) and mean (light line) temperature obtained with the fine (dashed line) and homogenised (dot marker) model. (b) Resistance ratio $R_{\mathrm{AC}} / R_{\mathrm{DC}}$ obtained with the fine (dashed line) and homogenised (dot marker) model at different working conditions

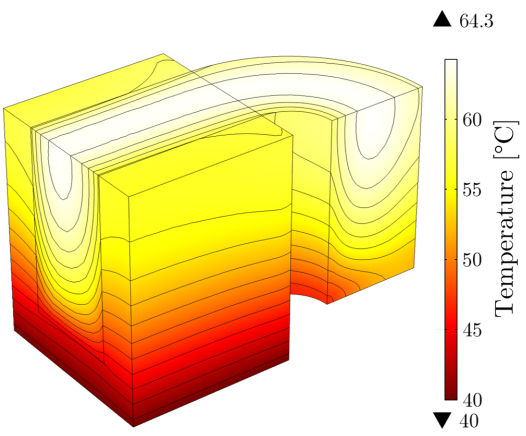

FIG. 6: Steady-state temperature distribution resulting from a DC load of $I=50 \mathrm{~A}$

TABLE I: REFERENCE MATERIAL PROPERTIES

\begin{tabular}{lll} 
Material & Variable & Value \\
\hline & $\rho_{0}$ & $1.76 \times 10^{-8} \Omega \mathrm{m}$ \\
Conductor: & $\alpha$ & $0.003751 / \mathrm{K}$ \\
copper & $k_{c}$ & $400 \mathrm{~W} / \mathrm{mK}$ \\
& $c_{c}$ & $386 \mathrm{~J} / \mathrm{kgK}$ \\
& $d_{c}$ & $8890 \mathrm{~kg} / \mathrm{m}^{3}$ \\
\hline Insulation: & $k_{i}$ & $0.26 \mathrm{~W} / \mathrm{mK}$ \\
dual coat & $c_{i}$ & $1000 \mathrm{~J} / \mathrm{kgK}$ \\
polyester grade 2 & $d_{i}$ & $1440 \mathrm{~kg} / \mathrm{m}^{3}$ \\
\hline Impregnation: & $k_{f}$ & $0.7 \mathrm{~W} / \mathrm{mK}$ \\
Epoxylite EIP & $c_{f}$ & $1700 \mathrm{~J} / \mathrm{kgK}$ \\
4260 & $d_{f}$ & $1766 \mathrm{~kg} / \mathrm{m}^{3}$ \\
\hline & $k_{\text {core } x, y}$ & $22 \mathrm{~W} / \mathrm{mK}$ \\
Core: & $k_{\text {core } z}$ & $2 \mathrm{~W} / \mathrm{mK}$ \\
M270-35A & $c_{\text {core }}$ & $460 \mathrm{~J} / \mathrm{kgK}$ \\
& $d_{\text {core }}$ & $7650 \mathrm{~kg} / \mathrm{m}^{3}$
\end{tabular}

analysis is to enable the accurate evaluation of the ratio $R_{\mathrm{AC}} / R_{\mathrm{DC}}$ usually employed to describe $\mathrm{AC}$ loss contribution, where $R_{\mathrm{AC}}=P_{t o t} / I_{r m s}^{2}$ with $P_{t o t}$ total winding loss. In fact, Fig. 5(b) clearly shows how the AC contribution is strictly dependent on the load: an higher current leads to higher temperatures, and accordingly increased electrical resistance, and this reduces the AC effects.

We conclude this section presenting Fig 6, where the temperature distribution due to a DC load is depicted. Here we want to highlight how the thermal hot spot not always is located in the end-winding region. Indeed, the large AC losses concentrated close to the slot opening lead the hot spot to move to the middle of the active region, notwithstanding the worse cooling conditions of the end-winding.

\section{B. Experimental validation}

The motorette described in the previous sections was built to validate the coupled homogenisation technique, see Fig. 7(a) where the motorette is shown before encapsulating the windings with epoxy. The material properties are listed in Table I. In particular, the insulation coating thickness was takes as $0.25 \mathrm{~mm}$ and the thermal conductivity of the epoxy compound 
was given by the manufacturer. The anisotropic thermal conductivity of the core was assumed based on the experimental work provided by [20]; on the other hand, the $\mathrm{BH}$ curve was provided by the manufacturer. Between the winding and the core a thin layer of liner $\left(k_{\text {liner }}=0.195 \mathrm{~W} / \mathrm{m} \mathrm{K}\right.$ and thickness $0.025 \mathrm{~mm}$ ) was added.

As shown in Fig. 7(a), the winding were equipped with a large number of thermocouples (type $\mathrm{K}$ and type $\mathrm{T}$ ), to accurately monitor the thermal behaviour during the tests. Before encapsulation, several sensors were embedded in the core as well.

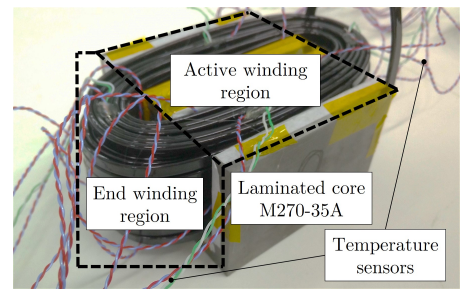

(a)

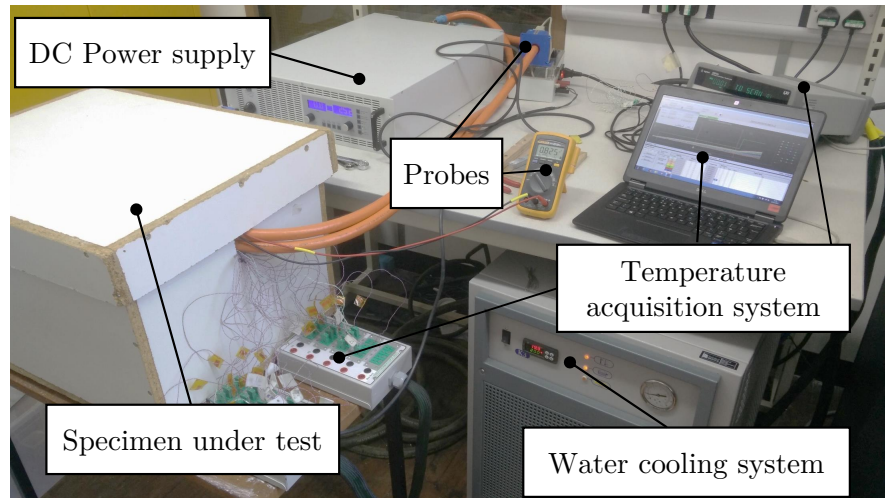

(b)

FIG. 7: (a) Motorette assembly before adding the thermocouples on the core and encapsulating the winding with epoxy and (b) experimental setup while performing DC tests

1) DC tests: To finalise the thermal model calibration, DC tests were first performed. As depicted in Fig. 7(b), the motorette was placed in a insulating chamber, suitably mounted on a liquid-cooled temperature-controlled plate. The winding and core temperatures were recorded while the motorette was loaded with the DC power supply. The test enabled to identify the build factors, including the thermal contact resistance between the winding and the core and between the motorette and the cooling plate. In the software we employed for this work, this can achieved adding a "thin layer" between the domains. In the model, a fixed temperature is imposed to the motorette base $\left(20^{\circ} \mathrm{C}\right)$, while on the remaining external boundaries no-flux condition is applied.

The results of the DC tests after the calibration are plotted in Fig. 8. In the figure we compare the measured and estimated temperatures within the winding compound. As described in Fig. 8(a), the thermocouples were placed in specific locations in the middle of the active and end winding regions: i.e. the

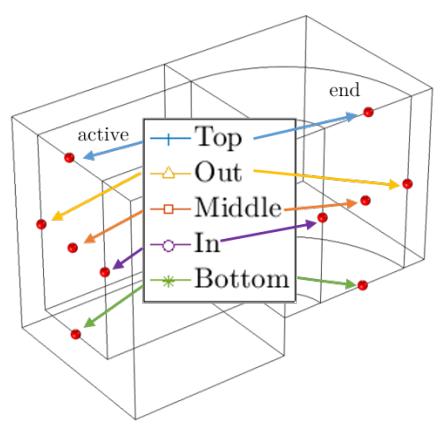

(a)

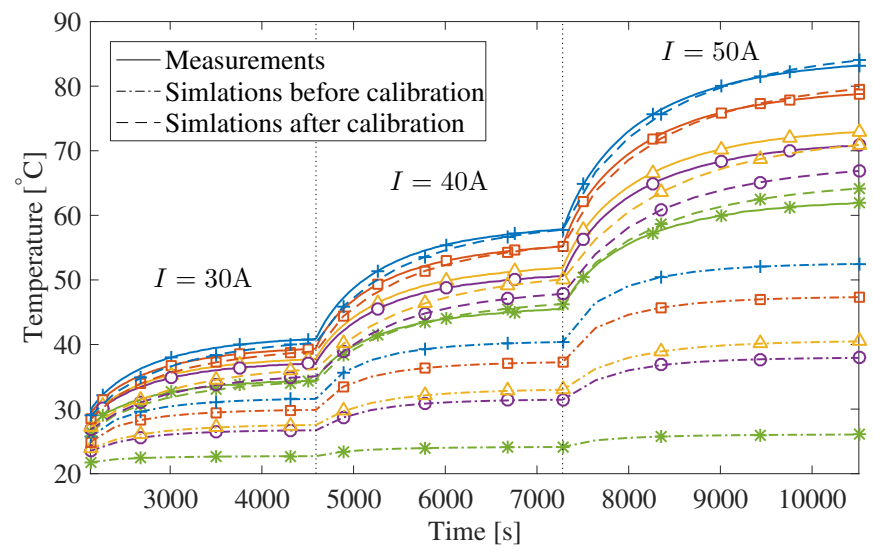

(b)

FIG. 8: (a) Sketch showing the locations where the sensors were placed in the winding. (b) Comparison between measured and estimated winding transient temperatures before and after calibration with motorette loaded with 30, 40 and $50 \mathrm{ADC}$ until thermal steady state is reached per each condition. Temperatures refer to the mid-cross section of the active windings

top, bottom, centre, inner and outer sides of the windings domain. The position of the sensors not only facilitates the calibration procedure, but also enables to obtain a good understanding of the temperature distribution within the slot. In Fig. 8(b) the temperature measured in the active-region mid-cross section are compared to the model estimations, before and after the calibration, over a transient where the load was switched when thermal steady state was reached. After the calibration a good matching is achieved in all the 5 locations, allowing to prove also the good quality of the applied thermal homogenisation. It is important to notice that without calibration, which are almost impossible to address analytically, we would have significantly underestimated the top temperatures.

2) AC tests: DC tests are used to calibrate various heat transfer effects, whereas the AC tests allow to identify potential issues associated with 'additional loss' due to manufacture, e.g. higher than expected core loss. To perform the AC tests, the arrangement shown in Fig. 7(b) was kept, only changing the power supply. Each test consisted of injecting an AC sinusoidal load in the motorette until thermal steady state was reached (a convergence strategy of $\Delta T<1^{\circ} \mathrm{C}$ over the last $10 \mathrm{~min}$ was applied) and then the real power was 


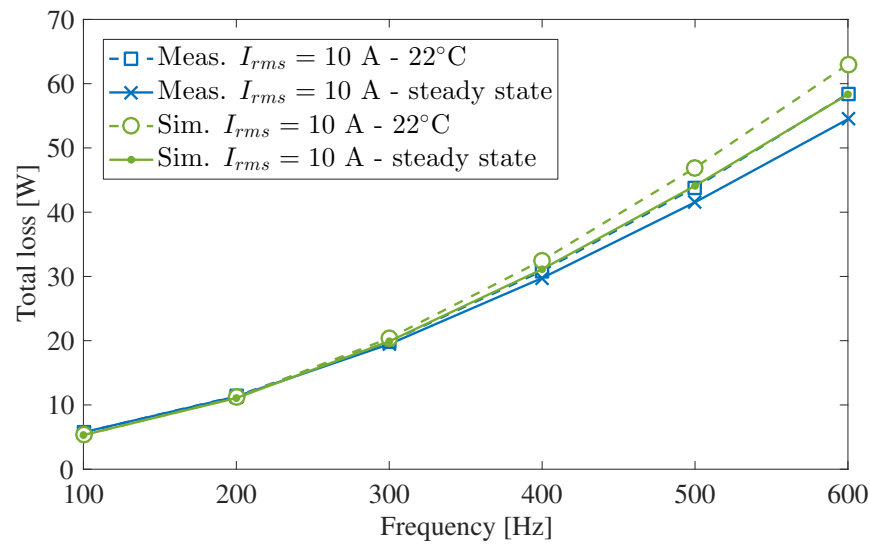

(a)

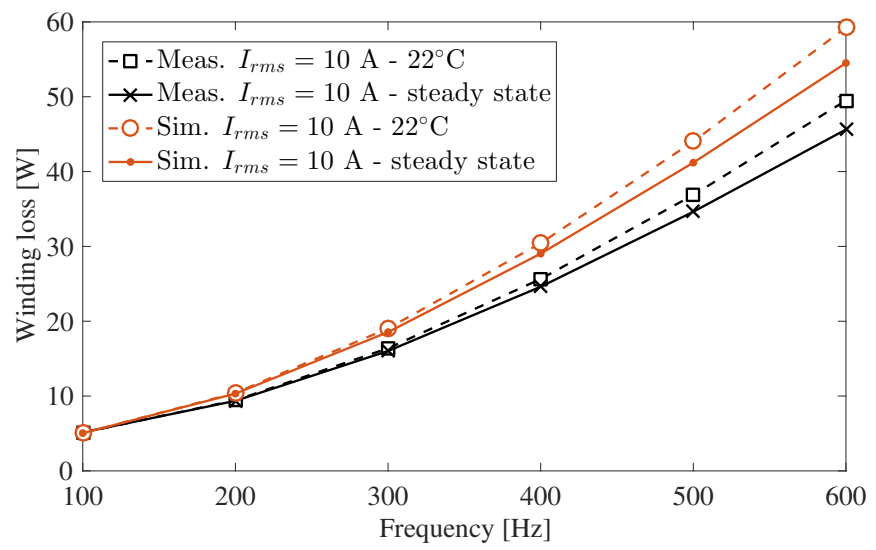

(b)

FIG. 9: Predicted and measured (a) total (including core) and (b) winding loss with the motorette loaded with $I_{r m s}=10 \mathrm{~A}$ and $f=100 \rightarrow 600 \mathrm{~Hz}$ at $22{ }^{\circ} \mathrm{C}$ (dashed line) and at thermal steady state (solid line)

measured with the help of a power analyser. The core losses were previously evaluated on separate tests. The losses were then compared to the case without temperature effects, that is injecting the AC load for few seconds avoiding the windings temperature to change significantly.

These results due to a load of $I_{r m s}=10 \mathrm{~A}$ with the frequency ranging from $100 \mathrm{~Hz}$ to $600 \mathrm{~Hz}$ are compared in Fig. 9, where with the dash line are highlighted the total loss at ambient temperature $\left(22^{\circ} \mathrm{C}\right)$ and with the solid line the measurements and simulations at thermal steady state. On the other hand, in Fig. 9(b), we compare the winding loss, That is removing the core contribution to the loss. The coupled electromagnetic-thermal model slightly overestimates the winding loss, with an error of $\approx 20 \%$ compared to the measured values. The core losses are instead underestimated by the model, balancing the winding loss discrepancy. Accordingly, the simulated total loss follow closer the measurements. In general, predictions follow the same loss trend as measurements and, more importantly, the winding loss reduction due to thermal effects is captured with good accuracy. Part of this error is systematic and due to homogenisation, as shown in the numerical validation. Other discrepancies may be due

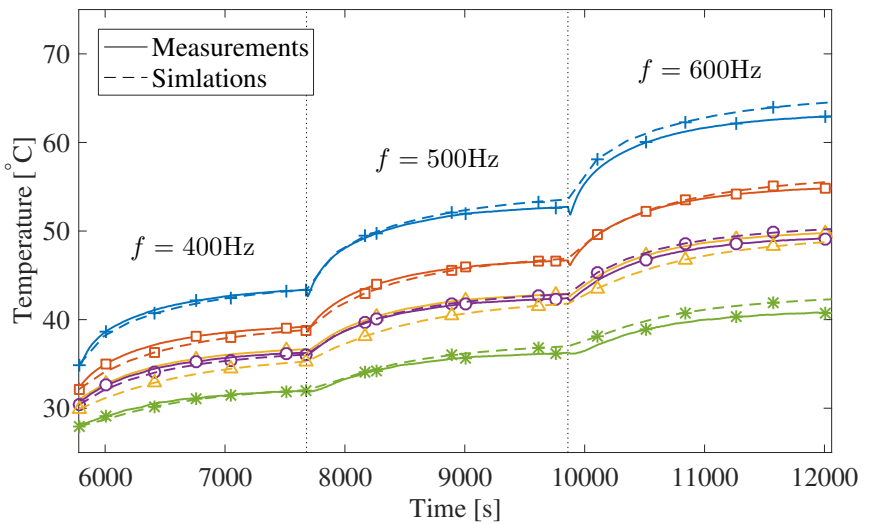

FIG. 10: Comparison between measured and estimated winding transient temperatures after calibration with motorette loaded with $I_{r m s}=10 \mathrm{~A}$ at 400,500 and $600 \mathrm{~Hz}$ until thermal steady state is reached per each condition. Temperatures refer to the mid-cross section of the active windings

to building factors that cannot be captured by the model or measurements intrinsic errors.

It is now interesting to check the accuracy of the proposed model in the prediction of the temperature distribution within the slot. Similarly to Fig. 8, in Fig. 10 we compare temperatures in the same five locations within the mid-cross section of the active winding region. The model is able to accurately predict the large thermal gradient developing in the slot not only at steady state, but also during the whole transient. Compared to the DC test, we can clearly notice how the particular loss distribution, concentrated in the neighbourhood of the slot opening, leads to a significantly steeper temperature gradient. As an example we can consider the temperature distributions due to a similar amount of power loss in DC or AC. In the first case - $P=58 \mathrm{~W}$ with $I=40 \mathrm{~A} \mathrm{DC} \mathrm{-} \mathrm{the}$ temperature difference measured between the top and bottom of slot is $\Delta T_{\mathrm{DC}}=12{ }^{\circ} \mathrm{C}$ as shown in Fig. 8; on the other hand, the gradient when a current of amplitude $I_{r m s}=10 \mathrm{~A}$ at $600 \mathrm{~Hz}$ is injected $(P=50 \mathrm{~W})$ was $\Delta T_{\mathrm{AC}}=22^{\circ} \mathrm{C}$ - see Fig. 10.

In Table II we compare the steady-state top temperatures in the active and end regions obtained through measurements or estimated by the model. The agreement is very good, with the error falling below $3{ }^{\circ} \mathrm{C}$. The measurements seem not to respect one of the outcomes of section III-A, since we recorded the highest temperature always in the active region, for both $\mathrm{AC}$ and DC tests. It must be said, however, that according to Table II for DC test the $\Delta T$ between end and active top temperature was $<1.5^{\circ} \mathrm{C}$, while with the inclusion of AC losses, the difference was always $>1.5^{\circ} \mathrm{C}$, with a peak of $3.1^{\circ} \mathrm{C}$.

\section{Conclusions}

In this work a method was presented to significantly reduce the computational effort for 3D electromagnetic-thermal coupled FE analysis via homogenisation. Compared to the solution of a fine model, where every single wire is taken into account, the results presented here show that the homogenised 
TABLE II: TOP TEMPERATURES IN ACTIVE AND END WINDING REGIONS WITH DC AND AC

\begin{tabular}{l|ccc|ccc}
\multirow{2}{*}{ Current } & \multicolumn{3}{c}{ Active winding top temperature } & \multicolumn{3}{c}{ End winding top temperature } \\
& Meas. $\left[{ }^{\circ} \mathrm{C}\right]$ & Sim. $\left[{ }^{\circ} \mathrm{C}\right]$ & $\Delta T_{\text {S-M }}\left[{ }^{\circ} \mathrm{C}\right]$ & Meas. $\left[{ }^{\circ} \mathrm{C}\right]$ & Sim. $\left[{ }^{\circ} \mathrm{C}\right]$ & $\Delta T_{\text {S-M }}\left[{ }^{\circ} \mathrm{C}\right]$ \\
\hline$I=30 \mathrm{~A} \mathrm{DC}$ & 40.7 & 40.3 & -0.4 & 40.4 & 40.4 & 0 \\
$I=40 \mathrm{~A} \mathrm{DC}$ & 57.6 & 57.8 & 0.2 & 56.8 & 58 & 2.2 \\
$I=50 \mathrm{~A} \mathrm{DC}$ & 83.2 & 84 & 0.8 & 81.7 & 84.4 & 2.7 \\
\hline$I_{r m s}=5 \mathrm{~A}$ at $900 \mathrm{~Hz}$ & 41.4 & 41.9 & 0.5 & 39.9 & 40.6 & 0.7 \\
$I_{r m s}=5 \mathrm{~A}$ at $1000 \mathrm{~Hz}$ & 44.7 & 45.5 & 0.8 & 43 & 44 & 1 \\
$I_{r m s}=5 \mathrm{~A}$ at $1100 \mathrm{~Hz}$ & 48.7 & 48.9 & 1.2 & 45.9 & 47.3 & 1.4 \\
\hline$I_{r m s}=10 \mathrm{~A}$ at $400 \mathrm{~Hz}$ & 43.2 & 43.4 & 0.2 & 41.6 & 42.1 & 0.5 \\
$I_{r m s}=10 \mathrm{~A}$ at $500 \mathrm{~Hz}$ & 52.6 & 53.6 & 1 & 50.3 & 51.7 & 1.4 \\
$I_{r m s}=10 \mathrm{~A}$ at $600 \mathrm{~Hz}$ & 62.9 & 64.5 & 1.6 & 59.8 & 62.1 & 2.3
\end{tabular}

model is able to provide accurate estimations. In particular, the method showed to be suitable for loss separation (active vs. end winding loss contribution) thanks to the reduced model complexity. The method, here applied to a reference stator-winding assembly, can be applied to any electromagnetic device, such as transformers or electrical machines. The numerical results were confirmed by the measurements performed on a suitably built testing exemplar.

From the results, the following conclusions can be drawn: (i) temperature effects significantly affect the ratio $R_{\mathrm{AC}} / R_{\mathrm{DC}}$. This confirms that a coupled electromagnetic-thermal analysis, even at design stage, is needed to obtain a more accurate evaluation of the specimen performance. (ii) the effect of AC losses, concentrated near the slot opening, increases the temperature gradient within the winding domain. This means that, in such conditions, the winding mean temperature can be a too reductive information to describe the winding thermal behaviour. Indeed precise estimations of the actual thermal field can help provide more reliable predictions of the conductor insulation lifespan. (iii) due to the contribution of AC effects, a significant gradient between end and active winding top temperatures is expected, with the hotspot located in the active region.

It is worth noting that the electromagnetic problem winding domain cannot be homogenised when the current flows in parallel path, since in general there will be a difference in the current carried by each conductor. When applied to electrical machines, however, this issue can be overcome if the conductors are properly transposed, reducing circulating currents [21]. On the other hand, the limit of the thermal homogenisation lays on the winding impregnation. When the windings are not impregnated, i.e. the filling of the slot is air, the heat transfer is not driven by conduction only. This significantly complicates the modelling of this phenomenon, which can longer easily homogenised.

As a final remark, compared to the fine model, the homogenised model requires some pre-calculations to obtain the effective material properties, which are not computationally demanding. The homogenised model can then be implemented in most of the commercial (as in this work) or open-source FE software. Accordingly, compared to other analytical methods, this approach requires none of very little coding.

\section{REFERENCES}

[1] A. Boglietti, S. Member, A. Cavagnino, D. Staton, M. Shanel, M. Mueller, and C. Mejuto, "Evolution and Modern Approaches for Thermal Analysis of Electrical Machines," IEEE Transactions on Industrial Electronics, vol. 56, no. 3, pp. 871-882, 2009.

[2] R. Wrobel, N. McNeill, and P. H. Mellor, "Performance Analysis and Thermal Modeling of a High-Energy-Density Prebiased Inductor," IEEE Transactions on Industrial Electronics, vol. 57, no. 1, pp. 201-208, 2010.

[3] S. Ayat, R. Wrobel, J. Goss, and D. Drury, "Experimental Calibration in Thermal Analysis of PM Electrical Machines," in IEEE Energy Conversion Congress and Exposition, (ECCE), 2016.

[4] R. Wrobel and N. Simpson, "Winding Loss Separation in Thermal Analysis of Electromagnetic Devices," in International Conference on Electrical Machine (ICEM), 2016, pp. 2135-2141.

[5] R. Wrobel, D. E. Salt, A. Griffo, N. Simpson, and P. H. Mellor, "Derivation and scaling of AC copper loss in thermal modeling of electrical machines," IEEE Transactions on Industrial Electronics, vol. 61, no. 8, pp. 4412-4420, 2014.

[6] P. Mellor, R. Wrobel, and N. Simpson, "AC Losses in High Frequency Electrical Machine Windings formed from Large Section Conductors," in Energy Conversion Congress and Exposition (ECCE), 2014, pp. 55635570.

[7] P. B. Reddy, Z. Q. Zhu, S. H. Han, and T. M. Jahns, "Strand-level proximity losses in PM machines designed for high-speed operation," in International Conference on Electrical Machines (ICEM), no. 1, 2008, pp. $1-6$.

[8] D. A. Gonzalez and D. M. Saban, "Study of the copper losses in a highspeed permanent-magnet machine with form-wound windings," IEEE Transactions on Industrial Electronics, vol. 61, no. 6, pp. 3038-3045, 2014.

[9] P. B. Reddy, T. M. Jahns, and T. P. Bohn, "Modeling and Analysis of Proximity Losses in High-Speed Surface Permanent Magnet Machines with Concentrated Windings," in Energy Conversion Congress and Exposition (ECCE), 2010, pp. 996-1003.

[10] J. Gyselinck and P. Dular, "Frequency-domain homogenization of bundles of wires in 2-D magnetodynamic FE calculations," IEEE Transactions on Magnetics, vol. 41, no. 5, pp. 1416-1419, 2005.

[11] M. El Feddi, Z. Ren, A. Razek, and A. Bossavit, "Hogenization Technique for Maxwell Equations in Periodic Structures," IEEE Transactions on Magnetics, vol. 33, no. 2, pp. 1382-1385, 1997.

[12] G. Meunier, V. Charmoille, C. Guérin, P. Labie, and Y. Maréchal, "Homogenization for periodical electromagnetic structure: Which formulation?" IEEE Transactions on Magnetics, vol. 46, no. 8, pp. 34093412, 2010.

[13] D. C. Meeker, "An improved continuum skin and proximity effect model for hexagonally packed wires," Journal of Computational and Applied Mathematics, vol. 236, no. 18, pp. 4635-4644, 2012. [Online]. Available: http://dx.doi.org/10.1016/j.cam.2012.04.009 
[14] P. Romanazzi, J. Gyselinck, M. Bruna, and D. A. Howey, "Electromagnetic and thermal homogenisation of an electrical machine slot," in International Conference on Electrical Machine (ICEM), Lausanne, 2016.

[15] R. V. Sabariego, P. Dular, and J. Gyselinck, "Time-domain homogenization of windings in 3-D finite element models," IEEE Transactions on Magnetics, vol. 44, no. 6, pp. 1302-1305, 2008.

[16] F. Chauvicourt, P. Romanazzi, D. Howey, A. Dziechciarz, C. Martis, and C. T. Faria, "Review of Multidisciplinary Homogenization Techniques applied to Electric Machines," in Eleventh International Conference on Ecological Vehicles and Renewable Energies (EVER), Monaco, 2016.

[17] P. Romanazzi, M. Bruna, and D. A. Howey, "Thermal homogenisation of electrical machine windings applying the multiple-scales method," ASME Journal of Heat transfer, vol. 139, no. January, 2017.

[18] O. Moreau, L. Popiel, and J. L. Pages, "Proximity losses computation with a 2D complex permeability modelling," IEEE Transactions on
Magnetics, vol. 34, no. 5 PART 1, pp. 3612-3615, 1998.

[19] R. Wrobel, A. Mlot, and P. H. Mellor, "Contribution of End-Winding Proximity Losses to Temperature Variation in Electromagnetic Devices," IEEE Transactions on Industrial Electronics, vol. 59, no. 2, pp. 848-857, 2012.

[20] J. E. Cousineau, K. Bennion, M. Mihalic, S. Narumanchi, J. E. Cousineau, K. Bennion, M. Mihalic, and S. Narumanchi, "Characterization of Contact and Bulk Thermal Resistance of Laminations for Electric Machines Characterization of Contact and Bulk Thermal Resistance of Laminations for Electric Machines," National Renewable Energy Laboratory (NREL), Golden, CO (United States), Tech. Rep. June, 2015.

[21] J. Gyselinck, P. Dular, N. Sadowski, P. Kuo-Peng, and R. Sabariego, "Homogenization of Form-Wound Windings in Frequency and Time Domain Finite-Element Modeling of Electrical Machines," IEEE Transactions on Magnetics, vol. 46, no. 8, pp. 2852-2855, 2010. 